\title{
A Tabu Search Algorithm for Determining the Economic Design Parameters of an Integrated Production Planning, Quality Control and Preventive Maintenance Policy
}

\author{
Abdur Rahim \\ Faculty of Business Administration \\ University of New Brunswick, Fredericton, NB, Canada \\ Muhammad Shakil \\ Department of Systems Engineering \\ King Fahd University of Petroleum and Minerals, Dhahran 31261, Saudi Arabia
}

\begin{abstract}
In this work, an integrated model of economic production quantity, economic design of an $\bar{x}$-control chart, and preventive maintenance are investigated under non-uniform quality control parameters. The effect on the expected total cost and quality control cost is investigated with three different assumptions of the quality control parameters. Using a tabu seach algorithm, the optimal values of quality control parameters, for different PM levels, are found. A non-uniform scheme for sampling frequency, sample size and control limit co-efficient provides lower cost as compared to schemes where only sampling frequency is taken as non-uniform.
\end{abstract}

\section{Keywords}

Tabu search algorithm, economic design parameters, production planning, quality control, preventive maintenance

\section{Introduction}

Quality control, production planning and maintenance policy are important factors in any manufacturing process. Effective integration of these factors will give a company a competitive edge of advantage in the global market. The customers' satisfaction might lead to an increased cost of a production process. The optimization of production cost is an important area of research. Economic production quantity, economic design of an $\bar{x}$-control chart, and preventive maintenance are the three main elements in production management. In the past, these aspects have been considered separately and different modifications were made in the individual models to achieve optimal production costs. In this work, an integrated model of economic production quantity, quality control, and preventive maintenance policy is studied. Optimal values of design parameters of the model are obtained using a tabu-search algorithm.

\section{System Operations and Assumptions}

The same process is assumed as in Rahim and Ben-Daya [1]. It is assumed that the process is producing a single item. The process is new (i.e., age is zero) at the start and it is in an in-control state. The process may shift to an outof-control state as the system ages. The time that the process shifts to an out-of-control state is a random variable. It follows a general distribution with increasing hazard rate. The product quality is inspected using an $\overline{\mathrm{x}}$-control chart.

The process is inspected at time intervals $t_{1}, t_{2}, t_{3}, \ldots, t_{m} . m$ is the number of quality inspection intervals in a production cycle. Preventive maintenance activities are scheduled at $l$ integer multiples of quality inspection intervals, thus preventive maintenance activities are carried out at $t_{1 l}, t_{2 l}, t_{3 l}, \ldots$ If $l=1$, it means the preventive maintenance activities are carried out at each inspection interval. If $l=2$, it means that the preventive maintenance activities are scheduled at each alternative inspection interval. The process production cycle ends with $m$ quality intervals or a true alarm, indicating the process is out-of-control. The process is restored to an as good as new state through maintenance. Demand is constant and all demands must be met. Classical economic production quantity (EPQ) model assumptions apply here. 


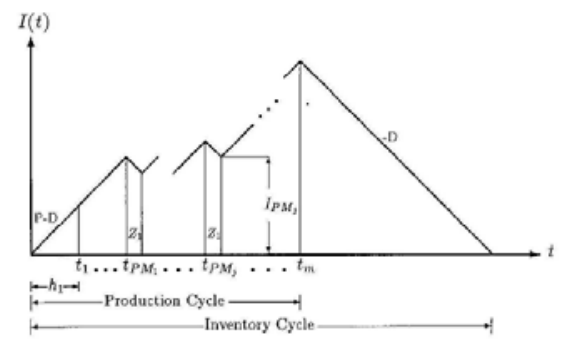

\section{Notations}

Figure 1: Inventory levels, $\mathrm{t}_{\mathrm{PMj}}$ is the times at the $\mathrm{j}$-th $\mathrm{PM}$

\subsection{Decision Variables}

Decision variables related to $\bar{x}$-control chart.

$m=$ Number of inspection intervals

$n_{j} \quad=$ Sample size at $j$-th inspection interval

$h_{j}=$ Sampling frequency at $j$-th inspection interval

$k_{j}=$ Control limit co-efficient at $j$-th inspection interval

Other notations for the $\overline{\mathrm{x}}$-control chart are as follows.

$Z_{1}^{\max }=$ Expected time to restore system to as good as new state

$Z_{1} \quad=$ Expected time to perform one PM action

$Z_{2}=$ Expected time to repair system when failure is detected

$a \quad$ = Fixed cost for sampling

$b=$ Variable cost of sampling

$c_{f} \quad=$ Cost of detecting a false alarm

$c_{n n}=$ Quality cost while producing in an in-control state

$C_{\text {wat }}=$ Quality cost while producing in an out-of-control state

$B=$ Magnitude of the shift in process mean

$\alpha_{j}=$ Probability that, exceeding control limits; process in-control: at $j$-th inspection interval

$\beta_{j} \quad=$ Probability that, not exceeding control limits; process out-of-control: at $j$-th inspection interval

$F(t)=$ Cumulative time to shift distribution

$\bar{F}(t)=1-F(t)$

$Z_{1 j}=$ Equal to $Z_{1}$ if PM is carried out at $j$-th inspection

$t_{j}=$ Time at the end of $j$-th PM inspection interval $t_{1}=h_{1}$ and $t_{j}=h_{j}+\Sigma_{i=1}^{j-t_{1}}\left(z_{1} i+h_{i}\right)$ where $j=1, \ldots, m$

$E(t)=$ Expected total length of production cycle including PM time

ETC = Expected total cost

$E(Q \odot)=$ Expected quality cost per production cycle including repair cost; PM cost not included

Notation used for EPQ model

$L(t)=$ Salvage value at time $t$

$D \quad=$ Demand rate units per unit time 


\section{Rahim and Shakil}

$$
\begin{array}{ll}
t_{m} & =\text { Production run } \\
P & =\text { Production rate units per unit time } \\
I(t) & =\text { Inventory level at time } t \\
C_{h} & =\text { Inventory holding cost per unit, per unit time } \\
S_{0} & =\text { Setup cost per production cycle } \\
E(T) & =\text { Expected length of production cycle } \\
E\left(T_{I}\right) & =\text { Expected length of inventory cycle } \\
E(H C) & =\text { Expected inventory holding cost per production cycle }
\end{array}
$$

Notation used for preventive maintenance

$$
\begin{aligned}
& C_{p m}^{0}=\text { Maximum system age reduction } \\
& C_{p m}=\text { Cost of actual PM activities } \\
& E(P M)=\text { Expected preventive maintenance cost per production cycle }
\end{aligned}
$$

\subsection{Integrated Model Assumptions}

The process assumptions are kept the same as those made in Rahim and Ben-Daya [1].

- Duration of an in-control period is assumed to follow an arbitrary probability distribution $f(t)$, having an increasing hazard rate $r(t)$, and cumulative distribution function $F(t)$.

- The preventive maintenance actions reduce the age of the system proportional to the cost of the maintenance.

- If there is no PM, then it means the time to search for an assignable cause is negligible.

- During the PM activity, it is assumed that production ceases for a time $Z_{1} \cdot Z_{1}=\left(C_{p m} / C_{p m}^{o}\right) Z_{1}^{\max }$

- The production ends either with a true alarm or at time $t_{m}$, whichever occurs first. If there is no true alarm up to time $t_{m-1}$ then the cycle is allowed to continue for additional time $h_{m}$. There is no cost of sampling and charting during $m$ - th interval. At time $t_{m}$ necessary maintenance or equipment replacement is done.

- During the inspection, if an out-of-control state is observed then production ceases until accumulated on hand inventory is depleted to zero. Otherwise, production continues.

- A salvage value is employed in the model, since the residual life beyond a certain age for the systems involving increasing hazard rate will be rather short.

- Failure rate, $r(t)$ of the system is decreased after each PM.

- The age reduction of the system is a function of the cost of the maintenance $C_{p m}$. Let $y_{j}\left(w_{j}\right)$ denote the effective age of the equipment right before (right after) the $j-$ th $\mathrm{P}$

$$
w_{j}=\left(1-\gamma_{j}\right) y_{j}
$$

Equation 1 gives the linear relationship between PM cost and age reduction of the system, where $\gamma_{j}=\eta^{j-1} C_{p m} / C_{p m}^{o}$ where $\eta(0<\eta \leq 1)$ is the imperfectness factor for the equipment.

$$
\text { For } j=1, \quad y_{1}=h_{1} \quad \text { and for } \quad j=2,3, \ldots, m, \quad y_{j}=w_{j-1}+h_{j} \text {. }
$$

\section{Non-uniform Sampling Frequency, Sample Size and Control Limit Co-efficient}

In this work, the $\bar{x}$-control chart parameters are also assumed to be nonuniform. As the process ages, the way sample size and control limit coefficients change, are selected from Ohta and Rahim [2]. 


\subsection{Non-uniform sampling frequency $\left(h_{4}\right)$}

Uniform sampling provides a constant integrated hazard rate $(r(t))$ for a Markovian shock model. Benerjee and Rahim [3] extended this fact to non-Markovian shock models by choosing the length of sampling intervals, such that the integrated hazard rate over each interval is the same as for all intervals,

$$
\int_{0}^{t_{1}} r(t) d t=\int_{i_{j}^{t+1}}^{t_{j}} r(t) d t, \quad r(t)=\frac{f(t)}{F(t)}
$$

If the time process remains in an in-control state and follows a Weibull distribution.

$$
\begin{aligned}
& f(t)=\lambda w t^{v-1} e^{-\lambda k^{v}} \\
& \Rightarrow F(t)=1-\theta^{-\lambda t^{v}}
\end{aligned}
$$

where $\lambda$ and $v$ are shape parameters for the Weibull distribution. Since failure rate is reduced. Equation 2 becomes

$$
\begin{gathered}
\int_{w_{j-1}}^{y_{j}} r(t) d t=\int_{0}^{h_{1}} r(t) d t, \quad j=2,3,4, \ldots \\
h_{\mathrm{i}}=\left\{w_{i-1}^{v}+h_{1}\right\}^{1 / v}-w_{j-1} \text { for } j=1,2,3,4 \ldots, m
\end{gathered}
$$

\subsection{Non-uniform sample size $\left(n_{j}\right)$}

Non-uniform sample size is selected such that the relative proportion of sample size to corresponding sampling interval is constant. That is, $n_{j} / h_{j}=n_{1} / h_{1}$. It means that sample size drawn per unit time in each sampling interval is constant. Using $h_{j}$ from Equation 6, an expression for non-uniform sample size $n_{j}$ is obtained,

$$
n_{j}=\text { integer }\left\{\left(n_{1} / h_{1}\right)\left(\left[w_{j-1}+h_{1}^{v}\right]^{1 / n}-w_{j-1}\right)\right\}
$$

4.3 Non-uniform control limit co-efficient $\left(k_{3}\right)$

$k_{j}$ is selected such that power of the control chart remains same in each interval.

$$
\Rightarrow k_{j}=z+\left(k_{1}-z\right) \sqrt{n_{j}} / \sqrt{n_{1}}
$$

\section{Expected Total Cost}

Expected total cost per unit of time is obtained by,

$$
E T C=\left(5_{0}+E(H C)+E(P M)+E(Q C)\right) E\left(T_{l}\right)
$$

Where $E\left(T_{I}\right)$ is given by,

$$
\begin{gathered}
E\left(T_{l}\right)=P D\left(E(T)-n_{\mathrm{pm}} Z_{1}\right) \\
n_{\mathrm{pm}}=E(P M) C_{\mathrm{pm}}
\end{gathered}
$$

5.1 Expected length of production cycle

$$
\begin{aligned}
E(T)= & Z_{2}\left\{\sum_{j=1}^{m-1}\left(F\left(y_{j}\right)-F\left(w_{j-1}\right)\right)+\bar{F}\left(w_{m-1}\right)\right\} \quad \text {, where } \quad Z_{j 1}=\left\{\begin{array}{cc}
Z_{1} \text { if } & j=l, 2 l, 3 l, \ldots . . \\
0 & \text { Otherwise }
\end{array}\right. \\
& +\sum_{j=1}^{m-1} Z_{j 1} \bar{F}\left(w_{j-1}\right)+\sum_{j=1}^{m} h_{j} \bar{F}\left(w_{j-1}\right) \\
& +\sum_{j=1}^{m-1} \beta_{j}\left(F\left(y_{j}\right)-F\left(w_{j-1}\right)\right) \\
& \times\left\{\sum_{i=j+1}^{m}\left(h_{i}+Z_{i 1}\right) \beta_{i}^{m-j-1}+h_{m} \beta_{j}^{m-j-1}\right\}
\end{aligned}
$$

\subsection{Expected preventive maintenance cost}

$$
\begin{aligned}
E(P M)= & \sum_{j=1}^{m-1} \bar{F}\left(w_{j}\right) \\
& +\sum_{j=1}^{m-1} \beta_{j} C_{p m j}\left(F\left(y_{i}\right)-F\left(w_{j-1}\right)\right) \quad \text {, where } \quad C_{p m j}=\left\{\begin{array}{cc}
C_{p m} \quad \text { if } & j=l, 2 l, 3 l, \ldots . . \\
0 & \text { Otherwise }
\end{array}\right. \\
& \times\left(1+\sum_{i=1}^{m-j-1} i\left(1-\beta_{i}\right) \beta_{i}^{i-1}\right. \\
& \left.+(m-j-1) \beta_{j}^{m-j-1}\right)
\end{aligned}
$$




$$
t_{m} \text { is calculated using } \quad t_{m}=h_{m}+\sum_{i=1}^{(m-1)}\left(Z_{1} i+h_{i}\right)
$$

\subsection{Expected quality control cost}

$$
\begin{aligned}
E(Q C)= & a+b n_{1}+\sum_{j=1}^{m-2}\left(a+b n_{j}\right) \bar{F}\left(w_{j}\right)+\sum_{j=1}^{m-2} \beta_{j}\left(a+b n_{j}\right) \times\left(\sum_{i=1}^{m-j-1} i\left(1-\beta_{i}\right) \beta_{i}^{m-j-1}\right. \\
& \left.+(m-j-1) \beta_{j}^{(m-j-1)}\right)+\left(C_{\text {in }}-C_{\text {out }}\right) \sum_{j=1}^{m} \int_{w_{j-1}}^{y_{i}} t f(t) d t+C_{\text {in }} \sum_{j=1}^{m} h_{j} \bar{F}\left(w_{j-1}\right) \\
& +\left(C_{\text {out }}-C_{\text {in }}\right) \sum_{j=1}^{m} y_{i}\left(F\left(y_{i}\right)-F\left(w_{j-1}\right)\right)+C_{a}\left\{\sum_{j=1}^{m}\left(F\left(y_{i}\right)-F\left(w_{j-1}\right)\right)+\bar{F}\left(y_{m}\right)\right\} \\
& +C_{\text {out }}\left(\sum_{j=1}^{m-1} \beta_{j}\left(F\left(y_{i}\right)-F\left(w_{j-1}\right)\right) \times \sum_{i=j+1}^{m} h_{i} \beta_{i}^{i-j-1}\right) \\
& +C_{f} \sum_{j=1}^{m-1} \alpha_{j} \bar{F}\left(w_{j}\right)-\bar{F}\left(y_{m}\right) L\left(t_{m}\right)
\end{aligned}
$$

\subsection{Expected inventory holding cost}

$$
\begin{aligned}
& \text { Let } I(t) \text { be the inventory at time } t . E(H C) \text { is given by, } \quad E(H C)=C_{h} \int_{0}^{T_{I}} I(t) d t \\
& E(A) \text { is the area under } I(t) \text { and can be calculated as follows; } \quad=C_{h} E(A)
\end{aligned}
$$

Let $I_{j}$ and $I_{m}$ be the inventory at the $j-$ th and $m$-th inspection intervals respectively.

$$
\begin{aligned}
& I_{j}=\left\{\begin{array}{ccc}
I_{j-1}+(P-D) h_{j}-D Z_{1} & \text { if } & j=1,2,3, \ldots . \\
I_{j-1}+(P-D) h_{j} & j=m
\end{array} \quad E(A)=\sum_{j=1}^{m} U{ }_{j} \bar{F}\left(w_{j-1}\right)+\sum_{j=1}^{m-1}\left(1-\beta_{j}\right) B_{j}\left(F\left(y_{i}\right)-F\left(w_{j-1}\right)\right)\right. \\
& +B_{m} \bar{F}\left(w_{j-1}\right)+\sum_{j=1}^{m-1} \beta_{j}\left(F\left(y_{i}\right)-F\left(w_{j-1}\right)\right) \\
& \text { where } B_{j} \text { and } U_{j} \text { are given by, } \vec{B}_{j}=\frac{i_{j}^{2}}{2 \boldsymbol{D}} \quad \times\left\{\sum_{i=j+1}^{m} \beta_{i} U_{i}+\beta_{j}^{m-j-1} B_{m}\right\}
\end{aligned}
$$

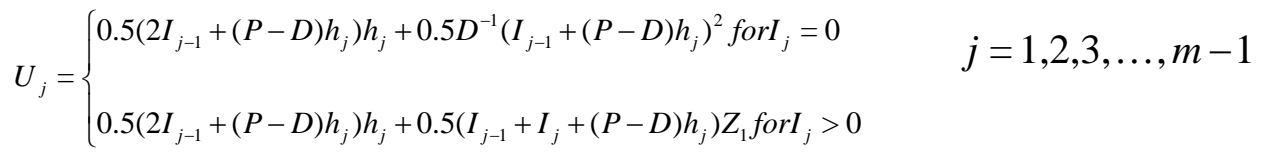

$$
\begin{aligned}
& U_{m}=0.5\left(2 I_{m-1}+(P-D) h_{m}\right) h_{m} \quad j=m
\end{aligned}
$$

\section{Optimal Parameter}

A problem is formulated in the form of Equation 9 containing five decision variables $h_{1}, k_{1}, n_{1}, m$ and $l . l$ is taken explicitly and different ETCs are found using different values of $l$. A tabu search algorithm in Glover and Taillard [4] is used here to find optimal solutions.

\section{Discussions}

Selection and non-uniform scheme for $h, n$ and $k$ is highly dependent on process type and operation. It is possible that selection of a non-uniform $n$ and $k$ does not have much effect on expected quality and total cost for certain processes. Consider the following cases:

\subsection{Case 1}

Consider a process manufacturing a single item; the process follows the same assumptions as made in section 2 . The item in production is expensive and requires destructive testing. So, the cost associated with taking a sample size ( $b$ ) is large. So selecting a non-uniform scheme for $n_{j}$ and $k_{j}$ (power of test will remain constant) for such processes will effect the $b n_{j}$ term. Expected total cost will be highly dependent on non-uniform sample size since, $n_{j}$ will be reduced in each quality inspection interval.

\subsection{Case 2}




\section{Rahim and Shakil}

Consider a process producing a automobile part on a automated CNC machine. Size and dimensions of such parts are controlled and checked through an automated process and requires much less time. In such cases, the cost associated with taking a sample is very small. So, selecting a non-uniform will have a very small effect on quality control costs, unless the part is examined in a separate quality department.

\section{Conclusion}

In this work the integrated model of production, quality and preventive maintenance was presented, where nonuniform sampling frequency, sample size, and control limit co-efficient were selected. Expected total and quality control cost was investigated for different preventive maintenance policies. Tabu search algorithm was used to find optimal values of system variables. It was found that a non-uniform scheme for sampling frequency, sample size and control limit co-efficient provided lower cost as compared to schemes where only sampling frequency is taken as non-uniform. It was also discussed that selection of a non-uniform sampling frequency and control limit co-efficient may not effect certain processes. Selection for such schemes is dependent on the process and operation under consideration.

\section{Acknowledgement}

Financial assistance of the National Sciences and Engineering Research Council of Canada and FDF grants from the Faculty of Business Adminstration, UNB, is gratefully acknowledged. The authors would also like to acknowledge King Fahd University of Petroleum and Minerals for facilitating this project. The assistance of Kim Wilson, Ralph O'Callaghan and Deyab Tamer, for typing, proof reading and editing the manuscript, is greatly appreciated.

\section{References}

1. Rahim, M.A, and Ben-Daya, M., 1998, “A Generalized Economic Model for Joint Determination of Production Run, Inspection Schedule, and Control Chart Design”, International Journal of Production Research, 36(1), 277-289.

2. Ohta, H., and Rahim, M.A., 1997, “A Dynamic Model for an $\overline{\mathrm{x}}$-Control Chart Design”, IIE Transactions, 29(6), 481-486.

3. Benerjee, P.K., and Rahim, M.A., 1988, "Economic Design of $\overline{\text { X}}$-Control Charts Under Weibull Shock Models”, Technometrics, 30(4), 407-414.

4. Glover, F., and Taillard, E., 1993, “A User's Guide to Tabu Search”, Annals of Operations Research, 41(1), 328. 